\title{
On the Connection between One- and Two-Equation Models of Turbulence
}

\author{
F. R. Menter" \\ Ames Research Center
}

\begin{abstract}
Summary
A formalism will be presented that allows the transformation of two-equation eddy viscosity turbulence models into one-equation models. The transformation is based on an assumption that is widely accepted over a large range of boundary layer flows and that has been shown to actually improve predictions when incorporated into two-equation models of turbulence. Based on that assumption, a new one-equation turbulence model will be derived. The new model will be tested in great detail against a previously introduced one-equation model and against its parent twoequation model.
\end{abstract}

\section{Introduction}

Since the emergence of sufficient computational resources and adequate computer codes to solve the Reynolds-averaged Navier-Stokes equations, the turbulence models of choice have been either algebraic models like the Baldwin-Lomax model (ref. 1), or two-equation eddy viscosity models. The main shortcoming of the algebraic models is the necessity to compute an algebraic lengthscale which becomes increasingly more difficult as the geometry and the flow fields become more complex. Furthermore, algebraic models fail to account for the important non-equilibrium effects and thereby consistently fail to predict the onset and amount of separation in adverse pressure gradient flows. The Johnson-King model (ref. 2) remedies this deficiency, by introducing a transport equation for the turbulent shear stress, but the model still depends on the specification of an algebraic length-scale.

Two-equation models have the advantage that they are independent of an algebraic length-scale and can therefore be applied to more complex flowfields. The models are built on the assumption that at least two scales are needed to describe the dynamics of turbulence. A number of different models has been proposed over the years. but they all have in common that a time-scale and a length-scale

\footnotetext{
"Research Scientist
}

can be extracted from the two independent variables. Among the two-equation models, the $\mathrm{k}-\varepsilon$ model (ref. 3 ) is most widely used in Reynolds-averaged Navier-Stokes computations. It solves one transport equation for the turbulent kinetic energy, $k$, and a second transport equation for the turbulent dissipation rate, $\varepsilon$. The model has been applied to a large number of flow problems and generally produced good results. The main shortcoming of the model is its inability to accurately predict adverse pressure gradient flows, as was found in the AFOSR Stanford conference in 1981/82 (ref. 4). Furthermore, the model can be numerically stiff when integrated through the sublayer and a significant increase of computing time can result from the necessity to reduce the time step in order to obtain converged solutions.

Recently the monopoly of two-equation models as the simplest complete (not depending on the specification of an algebraic length-scale (ref. 5)) models has been challenged by the re-emergence of one-equation turbulence models. While one-equation models have been used earlier (ref. 6), most of these older models solve an equation for the turbulent kinetic energy (or the turbulent shear stress) and still depend on the specification of an algebraic length-scale in order to calculate the eddy viscosity (see however references 7 and 8). The model introduced by Baldwin and Barth (ref. 9) solves one transport equation for the eddy viscosity and is independent of an algebraic length-scale. The Baldwin-Barth model is derived from the $k-\varepsilon$ model and a number of additional simplifying assumptions. However, in the course of the transformation, several diffusive terms are neglected and one diffusion term that does not follow from the transformation is introduced. The effect of changing these terms can not easily be apprehended beforehand, and it turns out that the Baldwin-Barth model does perform very differently from the underlying $k-\varepsilon$ model even for simple equilibrium flows. The change in the diffusive terms also changes the behavior of the model near the edge of shear layers and renders the equations ill-conditioned in that region as will be shown later. 
Due to these additional assumptions involved in the derivation of the Baldwin-Barth model, the connection between one- and two-equation turbulence models is not entirely clear and the use of one-equation models is often associated with a significant loss of generality.

The aim of the present effort is to establish a firm connection between one- and two-equation turbulence models that will enable the reader to make a judgement as to when the use of a one-equation model is appropriate (or even superior to a two-equation model). To achieve this goal, the $k-\varepsilon$ model will be transformed to a one-equation model based on a set of clearly defined assumptions. Numerical results based on the new model will be compared to those of the underlying k- $\varepsilon$ model and the Baldwin-Barth model. The computations will show that the new model performs very similar to the $k-\varepsilon$ model in situations were the underlying assumptions are acceptable. It will also be shown that the Baldwin-Barth model gives results very different from its parent $k-\varepsilon$ model.

It is to be emphasized that the purpose of the present work is to establish a clear connection between one- and twoequation models of turbulence and not to endorse a new model for general use. Due to the close relation to the standard $k-\varepsilon$ model, the new model inherits some of that model's deficiencies. Especially for aerodynamic flows, the new model does not perform as well as models specifically designed for these applications, like the one-equation model of Spalart and Allmaras (refs. 10 and 11) or the author's SST $k$ - $\omega$ model (refs. 12-14). It should be pointed out that the improved performance of these models is achieved by allowing the high Reynolds number form of the equations to explicitly depend on the distance from the surface. This additional degree of freedom allows for a more general calibration which can accommodate both, boundary layer as well as free shear layer flows more accurately. In contrast, the high Reynolds number form of the models tested here is strictly local, a feature that is very appealing from a computational standpoint.

This paper has greatly benefited from many important comments by Barrett Baldwin, Peter Bradshaw, Tom Coakley, and Philippe Spalart. I want to especially acknowledge Philippe Spalart, who brought to my attention the problems of the Baldwin-Barth model near the boundary layer edge.

\section{Turbulence Models}

\section{Transformation of the $k-\varepsilon$ Model}

This section will present a transformation of the high Reynolds number version of the $k-\varepsilon$ model to a one-equation model. For simplicity the equations will be written in boundary layer coordinates-the general form of the equations will be given later. The $k-\varepsilon$ model reads in boundary layer coordinates ( $x$-streamwise coordinate, $y$ normal to layer):

$$
\begin{aligned}
& \frac{D k}{D t}=\bar{v}_{t}\left(\frac{\partial u}{\partial y}\right)^{2}-\varepsilon+\frac{\partial}{\partial y}\left(\frac{\bar{v}_{t}}{\sigma_{k}} \frac{\partial}{\partial y}(k)\right) \\
& \frac{D \varepsilon}{D t}=c_{\varepsilon 1} \frac{\varepsilon_{k}}{k} \bar{v}_{t}\left(\frac{\partial u}{\partial y}\right)^{2}-c_{\varepsilon 2} \frac{\varepsilon^{2}}{k}+\frac{\partial}{\partial y}\left(\frac{\bar{v}_{t}}{\sigma_{\varepsilon}} \frac{\partial}{\partial y}(\varepsilon)\right)
\end{aligned}
$$

with the following definition for the eddy viscosity:

$$
\tilde{v}_{t}=c_{\mu} \frac{k^{2}}{\varepsilon}
$$

In order to arrive at a one-equation model, we follow Baldwin and Barth and express the time derivative of the eddy viscosity by the time derivatives of $k$ and $\varepsilon$ :

$$
\frac{D \tilde{v}_{t}}{D t}=c_{\mu}\left(2 \frac{k}{\varepsilon} \frac{D k}{D t}-\frac{k^{2}}{\varepsilon^{2}} \frac{D \varepsilon}{D t}\right)
$$

Replacing the total derivatives of $k$ and $\varepsilon$ on the right hand side by the right hand sides of equation 11 gives a single transport equation for the eddy viscosity, which, however, depends on $k$ and $\varepsilon$ as well as on the eddy viscosity:

$$
\frac{D \bar{v}_{s}}{D t}=F\left(\tilde{v}_{\imath} ; k ; \varepsilon\right)
$$

This presents a closure problem with one equation for three unknowns. In order to close the equation, two additional relations have to be provided. The first one is the definition of the eddy viscosity, equation 2 , which allows one to replace $\varepsilon$ by the eddy viscosity and the turbulent kinetic energy:

$$
\varepsilon=c_{\mu} \frac{k^{2}}{\bar{v}_{t}}
$$


with $c_{\mu}=0.09$. Note that this relation does not involve any assumptions and the resulting equation is still equivalent to the original $k-\varepsilon$ model. A second relation is needed to eliminate $\mathrm{k}$ from the right hand side of equation 4 and this relation can obviously not be derived from the $k-\varepsilon$ model (otherwise the $k-\varepsilon$ equations would be overspecified). However, there is a relation readily available, that relates the turbulent kinetic energy and the eddy viscosity, which has been confirmed for a large number of experimental boundary layer data and was used by Bradshaw et al. (ref. 6) in the derivation of their one-equation model, namely (see also ref. 15):

$$
\left|-\overline{u^{\prime} v^{\prime}}\right|=\bar{v}_{t}\left|\frac{\partial u}{\partial y}\right|=a_{1} k
$$

where $a_{1}$ is a constant and $\left|\overrightarrow{u^{\prime} v}\right|$ is the turbulent shear stress. It has been shown in (refs. 11-14) that Bradshaw's relation (eq. et6), when introduced into the $k-\omega$ model leads to a significant improvement of predictions for adverse pressure gradient flows. Note that the relation between the turbulent shear stress and the turbulent kinetic energy that results from standard two-equation models is:

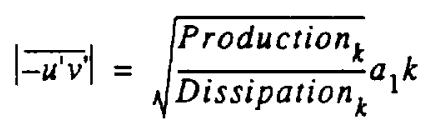

using $a_{1}=\sqrt{c_{\mu}}$. Equation 7 is not confirmed by experimental evidence. It is therefore to be expected that the introduction of equation 6 will actually lead to improved predictions of non-equilibrium flows.

Since we have a complete set of equations, the one-equation model can be derived by straightforward substitution. The result is:

$$
\begin{aligned}
\frac{D \bar{v}_{t}}{D t}= & c_{1} \bar{v}_{t}\left|\frac{\partial u}{\partial y}\right|-c_{2} \tilde{v}_{t}^{2}\left(\frac{\frac{\partial}{\partial y}\left|\frac{\partial u}{\partial y}\right|}{\left|\frac{\partial u}{\partial y}\right|}\right)^{2}+\frac{\partial}{\partial y}\left(\frac{\tilde{v}_{t}}{\sigma_{\varepsilon}} \frac{\partial}{\partial y}\left(\bar{v}_{t}\right)\right) \\
& +2 \frac{\left(\sigma_{\varepsilon}-\sigma_{k}\right)}{\sigma_{k} \sigma_{\varepsilon}}\left(\tilde{v}_{t} \frac{\partial^{2} \tilde{v}_{t}}{\partial y^{2}}+\left(\frac{\partial \bar{v}_{t}}{\partial y}\right)^{2}+3 \tilde{v}_{t} \frac{\partial \bar{v}_{t}}{\partial y}\left(\frac{\frac{\partial}{\partial y}\left|\frac{\partial u}{\partial y}\right|}{\left|\frac{\partial u}{\partial y}\right|}\right)\right. \\
& \left.+\tilde{v}_{t}^{2} \frac{1}{\left|\frac{\partial u}{\partial y}\right|} \frac{\partial^{2}}{\partial y^{2}}\left|\frac{\partial u}{\partial y}\right|\right)
\end{aligned}
$$

Equation 8 is very complicated and difficult to solve numerically. However, the contribution of the terms in the last parenthesis of the equation is proportional to the difference in the diffusive coefficients of the $k$ - and the $\varepsilon$ equation. For a number of $k-\varepsilon$ models these coefficients are equal (ref. 16) and the whole term is exactly zero. The importance of this term can be evaluated by changing the diffusion constants in the underlying $k-\varepsilon$ model and investigate the influence on the solution. In the logarithmic portion of a boundary layer, the expression inside the parenthesis is exactly equal to zero, so that the investigation can be restricted to flows away from the surface. Three sets of free shear layer computations have been performed with different values for the diffusive coefficient $\sigma_{\varepsilon}$. Note that the calibration of the standard $k-\varepsilon$ model for a logarithmic layer requires a diffusive coefficient of $\sigma_{\varepsilon}=1.17$ instead of the originally proposed $\sigma_{\varepsilon}=1.3$ (with $k=0.41$ ). The results of the computations are given in table $1\left(\sigma_{k}=1\right.$.)

Table 1. Free shear layer spreading rates for the $k-\varepsilon$ model with different values of $\sigma_{\varepsilon}$

\begin{tabular}{lccc}
\hline \multicolumn{1}{c}{ Flow } & $\sigma_{\varepsilon}=1.30$ & $\sigma_{\varepsilon}=1.17$ & $\sigma_{\varepsilon}=1.00$ \\
\hline Far wake & 0.255 & 0.256 & 0.257 \\
Mixing layer & 0.099 & 0.100 & 0.102 \\
Plane jet & 0.109 & 0.109 & 0.110 \\
Round jet & 0.120 & 0.121 & 0.124 \\
\hline \hline
\end{tabular}

It is obvious that changes in the diffusion coefficient $\sigma_{\varepsilon}$ have only limited influence on the computed results (the same is tue for $\sigma_{k}$ ). A $30 \%$ change in $\sigma_{\varepsilon}$ leads to a change of only $3 \%$ in the spreading rates. The assumption that $\sigma_{k}=\sigma_{\varepsilon}$ is therefore not very restrictive and the corresponding terms in equation 8 can be neglected. The second assumption in the derivation of the model is therefore:

$$
\sigma_{k}=\sigma_{\varepsilon} \equiv \sigma
$$

The resulting high Reynolds number form of the equation is:

$$
\frac{D \tilde{v}_{t}}{D t}=c_{1} \tilde{v}_{t}\left|\frac{\partial u}{\partial y}\right|-c_{2} \frac{\bar{v}_{t}^{2}}{L^{2} V K}+\frac{\partial}{\partial y}\left(\frac{\tilde{v}_{t}}{\sigma} \frac{\partial}{\partial y}\left(\tilde{v}_{t}\right)\right)
$$

Equation 10 involves the inverse of the v. Karman similarity length-scale $L_{V K}$ : 


$$
\frac{1}{L_{V K}}=\frac{\frac{\partial}{\partial y}\left|\frac{\partial u}{\partial y}\right|}{\left|\frac{\partial u}{\partial y}\right|}
$$

The v. Karman length-scale was not very successful when used in a mixing length model, mainly because it is singular whenever the denominator goes to zero. In the present one-equation model, the singularity (of the inverse of $L_{\mathrm{VK}}$ ) is not a problem, because the destruction term that involves $L_{\mathrm{VK}}$ can be limited by any other term that has the same dimension, as will be shown later. In the framework of eddy viscosity transport models, the v. Karman length scale was also utilized in an unpublished model by Baldwin (ref. 18), and in a model by Durbin et al. (ref. 19), but was never formally derived from the $k-\varepsilon$ model.

The coefficients of the new model follow directly from the $k-\varepsilon$ model constants:

$$
\begin{aligned}
& c_{1}=\left(c_{\varepsilon 2}-c_{\varepsilon 1}\right) \sqrt{c_{\mu}}=0.144 ; \quad \sigma=\sigma_{k}=1 \\
& c_{2}=\frac{c_{1}}{\kappa^{2}}+\frac{1}{\sigma}=1.86
\end{aligned}
$$

The standard $k-\varepsilon$ model constants of $c_{\varepsilon 1}=1.44, c_{\varepsilon 2}=1.92$, $c_{\mu}=0.09=a_{1}{ }^{2}$ and $\sigma_{k}=1.0$ have been used. Note that the transformation leads to $c_{2}=2 / \sigma_{\varepsilon}=1.71$ with $\sigma_{\varepsilon}=1.17$. Since $\sigma$ was chosen to be equal to $\sigma_{k}$ and not equal to $\sigma_{\varepsilon}$, the coefficient $c_{2}$ had to be slightly recalibrated to match the law of the wall.

The key to the understanding of the new model lies in the comparison of equations 6 and 7. For equilibrium flows the two formulations are equivalent and the one-equation model will be very close in performance to the $k-\varepsilon$ model. For non-equilibrium adverse pressure gradient flows, Bradshaw's relation, equation 6 , is actually better confirmed by experiments than equation 7 . For these flows the ratio of Production/Dissipation becomes larger than one in the outer region of the boundary layer and the k- $\varepsilon$ model will give higher shear stresses than the new model. Since the $k-\varepsilon$ model is well known to overpredict shear stress levels for these flows, it is to be expected that the new model will lead to improved predictions. For flows without shear, Bradshaw's relation, equation 6, has no meaning, and the new model cannot be expected to give good results. An example is isotropically decaying turbulence, where one-equation models predict that the eddy viscosity stays constant, whereas the $k-\varepsilon$ model predicts, more realistically, a decay of the turbulent variables. This deficiency is associated with the lack of a second scale in the model.
For shear flows the second scale is provided by the mean shear rate. Regions where the mean shear is locally zero are bridged by the diffusion and the convection terms.

Note however, that the overwhelming majority of applications of turbulence models is for shear flows, for which one-equation models are well suited.

The main assumption in the derivation of the new model is that the turbulent shear stress is proportional to the turbulent kinetic energy. In standard two equation models this assumption is equivalent to:

$$
\text { Production }_{k}=\text { Dissipation }_{k}
$$

used in the derivation of the Baldwin-Banth model. However. in that model only the production and dissipation terms are transformed based on equation 13. The diffusion terms are not transformed exactly. The high Reynolds number form of the Baldwin-Barh (BB) model is given by:

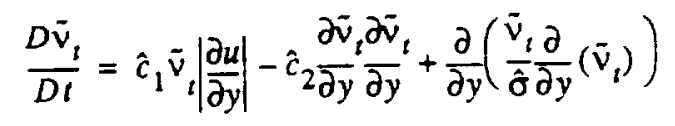

The original Baldwin-Barth model solves an equation for the turbulent Reynolds number. In order to allow a one-toone comparison of the constants, it has been reformulated here (exactly) as an equation for the eddy viscosity. The transformed constants for this model are:

$$
\begin{aligned}
& \hat{c}_{1}=\left(c_{\varepsilon 2}-c_{\varepsilon 1}\right) \sqrt{c_{\mu}}=0.24 ; \quad \hat{\sigma}=\sigma_{\varepsilon}=0.7 \\
& \hat{c}_{2}=\frac{\hat{c}_{1}}{\boldsymbol{\kappa}^{2}}+\frac{1}{\hat{\sigma}}=2.86
\end{aligned}
$$

They are based on $c_{\varepsilon 1}=1.2, c_{\varepsilon 2}=2.0, c_{\mu}=0.09=\mathrm{a}_{1}{ }^{2}$ and $\sigma_{\varepsilon}=0.7$ for the underlying $k-\varepsilon$ model. The low Reynolds number form of this model can be found in (ref. 9) and is not repeated here.

In order to distinguish the new model, equation 10 , from the other models in this study, we call it $(k-\varepsilon)_{1 E}$ model where the subscript stands for one-equation. The main difference between the $(k-\varepsilon)_{1 E}$ and the BB model is the form of the destruction term ( $c_{2}$ term). Although the derivation of the BB model starts out from the $k-\varepsilon$ model, the destruction term of that model does not follow from the transformation. The link between the BB model and the $k-\varepsilon$ model is broken by the introduction of this term and it is for this reason that the two models perform very differently. The influence of this term on predicted results will be shown later. 


\section{Low Reynolds Number Terms}

The assumptions leading to the $(\mathrm{k}-\varepsilon)_{1 \mathrm{E}}$ model are obviously not correct in the viscous sublayer, so that the low Reynolds number terms of the $k-\varepsilon$ model cannot be carried over to the one-equation model. This is not a great loss. because the near wall terms of the $k-\varepsilon$ model are generally complicated and difficult to integrate so that a one-to-one transformation is not desirable.

The purpose of damping functions is to reduce the eddy viscosity in the sublayer. In the present model this is achieved by reducing the production term as the wall is approached and by multiplying the high Reynolds number eddy viscosity, $\bar{v}_{t}$, by a damping function in order to arrive at the corrected eddy viscosity, $v_{l}$. The damping functions are designed in a pragmatic way that ensures that the resulting model is numerically stable and does not require excessive grid resolution near the surface. The present author does not consider it important to match the exact asymptotic behavior of the eddy viscosity near the surface, since the eddy viscosity is much smaller than the molecular viscosity in the immediate vicinity of the surface and has therefore no influence on the asymptotic form of the mean flow variables. Two damping functions are introduced, $D_{1}$ in front of the production term and $D_{2}$ into the definition of the eddy viscosity:

$$
\begin{aligned}
c_{1} \tilde{v}_{t}\left|\frac{\partial u}{\partial y}\right| & \rightarrow D_{1} c_{1} \tilde{v}_{t}\left|\frac{\partial u}{\partial y}\right| \\
v_{t} & =D_{2} \bar{v}_{s}
\end{aligned}
$$

based on the following expressions:

$$
\begin{aligned}
& D_{1}=\frac{v_{t}+v}{\bar{v}_{t}+v} \\
& D_{2}=1-e^{-\left(\frac{\tilde{v}_{t}}{A^{*} \times v}\right)}
\end{aligned}
$$

and $K=0.41$. Furthermore the molecular viscosity is added into the diffusion term in analogy to the $k-\varepsilon$ model. The coefficient $\mathrm{A}^{+}$is equal to $\mathrm{A}^{+}=13.5$. The complete form of the equations is given in the next section.

\section{General Form of the Equations}

In order to arrive at an invariant formulation, all occurrences of the strain rate are replaced by the absolute value of the vorticity $\Omega$ :

$$
\left|\frac{\partial u}{\partial y}\right| \rightarrow \Omega
$$

As has been pointed out by Spalart (ref. 10), alternative formulations like $\sqrt{U_{i, j} U_{i, j}}$ are possible, but for thin shear flows there is little difference between them. The term involving the inverse of the v. Karman length-scale becomes:

$$
E_{k-\varepsilon} \equiv \bar{v}_{t}^{2}\left(\frac{1}{L_{V K}}\right)^{2}=\bar{v}_{t}^{2}\left(\frac{\frac{\partial \Omega}{\partial x_{j}} \frac{\partial \Omega}{\partial x_{j}}}{\Omega^{2}}\right)
$$

An alternative but numerically more expensive form would be:

$$
E_{k-\varepsilon}=\tilde{v}_{t}^{2}\left(\frac{1}{L_{V K}}\right)^{2}=\tilde{v}_{t}^{2}\left(\frac{\frac{\partial^{2} u_{i}}{\partial x_{j} \partial x_{j}} \frac{\partial^{2} u_{i}}{\partial x_{k} \partial x_{k}}}{\frac{\partial u_{s} \partial u_{s}}{\partial x_{l}} \frac{\partial x_{l}}{\partial x_{l}}}\right)
$$

Similarly, all $y$-derivatives are replaced by their complete invariant forms.

As has been pointed out previously, the inverse of the $v$. Karman length-scale can become singular whenever $\Omega$ goes to zero, leading to an infinite destruction term $E_{k-\varepsilon}$. In order to prevent this from happening, the destruction term is limited by a multiple of the Baldwin-Barth destruction term, $\mathrm{E}_{\mathrm{BB}}$ :

$$
E_{1 e}=c_{3} E_{B B} \tanh \left(\frac{E_{k-\varepsilon}}{c_{3} E_{B B}}\right)
$$

with a constant $c_{3}=7 . \mathrm{E}_{\mathrm{BB}}$ is defined as:

$$
E_{B B}=\frac{\partial \tilde{v}_{t}}{\partial x_{j}} \cdot \frac{\partial \tilde{v}_{t}}{\partial x_{j}}
$$

Equation 23 provides a smooth transition between the two formulations whenever $E_{k-\varepsilon}$ goes to infinity. For most of the flow $E_{k-\varepsilon} / c_{3} E_{B B}$ and the original formulation is recovered. A less smooth transition could be achieved by $E_{1 e}=\min \left(E_{k-\varepsilon} ; c_{3} E_{B B}\right)$. The numerical results are not sensitive to the constant $c_{3}$.

The final form of the equations is:

$$
\frac{D \tilde{v}_{t}}{D t}=c_{1} D_{1} \tilde{v}_{t} \Omega-c_{2} E_{1 e}+\frac{\partial}{\partial x_{j}}\left(\left(v+\frac{\tilde{v}_{t}}{\sigma}\right) \frac{\partial}{\partial x_{j}}\left(\bar{v}_{t}\right)\right)
$$




$$
v_{t}=D_{2} \bar{v}_{t}
$$

\section{Numerical Results}

\section{Free Shear Flows}

Self similar shear layers are very important test cases which allow to obtain insight into the performance of turbulence models, without the need for large computer resources. In this section the models will be tested against the standard free shear cases, namely a self-similar mixing layer, the plane and round jet and the self similar far wake. The equations are cast into self-similar form following Wilcox (ref. 5), resulting in the following two ordinary differential equations for the non-dimensional velocity $U$ and the non-dimensional eddy viscosity $\mathrm{N}$ :

$$
\begin{gathered}
v \frac{d U}{d \eta}-\frac{1}{\eta^{j}} \frac{d}{d \eta}\left[\eta^{j} N \frac{d U}{d \eta}\right]=S_{U} U \\
V \frac{d N}{d \eta}-\frac{1}{\eta^{j}} \frac{d}{d \eta}\left[\frac{1}{\sigma^{j}} \eta^{j} N \frac{d N}{d \eta}\right]=S_{N} N+c_{1}\left|\frac{d U}{d \eta}\right|-c_{2}\left(\frac{N}{L_{V K}}\right)^{2}
\end{gathered}
$$

with:

$$
\frac{1}{L_{V K}}=\frac{\frac{1}{\eta^{j}} \frac{d}{d \eta}\left(\eta^{j \frac{d U}{d \eta}}\right)}{\frac{d U}{d \eta}}
$$

where $j=1$ for the round jet and $j=0$ for the plane flows. The non-dimensional variables are defined as follows:

Self-similar mixing layer:

$$
\begin{aligned}
u(x, y) & =U_{1} U(\eta) \\
v_{t}(x, y) & =x U_{1} N(\eta) \\
\eta & =\frac{y}{x}
\end{aligned}
$$

$U_{1}$ is the velocity of the upper stream (the lower stream has velocity zero).

\section{Self-similar far wake:}

$$
\begin{aligned}
& u(x, y)=U_{\infty}-\sqrt{\frac{D}{\rho x}} U(\eta) \\
& v_{t}(x, y)=\frac{D}{\rho U_{\infty}} N(\eta)
\end{aligned}
$$

$$
\eta=y \sqrt{\frac{\rho U_{\infty}^{2}}{D x}}
$$

$$
D=2 \int_{0}^{\infty} \rho u\left(U_{\infty}-u\right) d y
$$

Self-similar jet:

$$
\begin{aligned}
u(x, y) & =\frac{J}{x^{(j+1) / 2}} U(\eta) \\
v_{t}(x, y) & =\sqrt{J} x^{(1-j) / 2} N(\eta) \\
\eta & =\frac{y}{x} \\
J & =\frac{\pi^{j}}{2} \int_{0}^{\infty} u^{2} y^{j} d y
\end{aligned}
$$

The coefficients in the equations can be obtained from reference 5 and equation $3\left(S_{N}=2 S_{k}-S_{\varepsilon}\right.$. ) Note that the coefficient $S_{k}$ in reference 5 should be $2 U$ for the round jet):

Table 2. Coefficients for free shear flows

\begin{tabular}{lccc}
\hline \hline \multicolumn{1}{c}{ Flow } & $S_{U}$ & $S_{N}$ & $V(\eta)$ \\
\hline Far wake & $1 / 2$ & 0 & $--\frac{1}{2} \eta$ \\
Mixing layer & 0 & $-U$ & $-\int_{0}^{m} U\left(\eta^{\prime}\right) d \eta^{\prime}$ \\
Plane jet & $1 / 2 U$ & $-1 / 2 U$ & $-\frac{1}{2} \int_{0}^{\eta} U\left(\eta^{\prime}\right) d \eta^{\prime}$ \\
Round jet & $\mathrm{U}$ & 0 & $-\frac{1}{\eta} \int_{0}^{\eta} U\left(\eta^{\prime}\right) \eta^{\prime} d \eta^{\prime}$ \\
\hline \hline
\end{tabular}

\section{Asymptotic Solution Near Shear Layer Edge}

The analysis of the asymptotic solution near the edge of turbulent layer is an important part of turbulence model evaluation. It is especially important to determine the sensitivity of the solution to changes in the freestream values specified for the turbulence variables outside the layer. Models with solutions that change significantly with the freestream conditions are not desirable, because the "correct" freestream conditions are not known in most applications. The problem has been analyzed in detail in reference 17 for two-equation eddy viscosity models. It was shown that the $k-\omega$ model has a severe dependency on the values specified for $\omega$ outside the layer. For example, the 
maximum eddy viscosity inside a self-similar wake can be increased by almost one order of magnitude by reducing the freestream value of $\omega$. It was also shown that the $k-\varepsilon$ model does not suffer from this ambiguity. There is no theory available to decide whether a model has a freestream sensitivity, but the existence of an asymptotic algebraic solution near the boundary layer edge seems to be at least a necessary condition for a model to be well conditioned.

The analysis does not depend on which shear flow is selected for the analysis, since the existence of an algebraic solution implies that the terms involving $S_{U}$ and $S_{N}$ decay faster than the other terms near the edge of the layer and can therefore be neglected. The new variable $\tilde{\eta}=\eta-\delta$ is introduced near the shear layer edge, $\delta$, and algebraic solutions of the form:

$$
\frac{d U(\tilde{\eta})}{d \tilde{\eta}}=A \tilde{\eta}^{\alpha} ; \quad N(\tilde{\eta})=B \tilde{\eta}^{\beta}
$$

are introduced into the equations. Straightforward algebra shows that the exponents for the $(k-\varepsilon)_{1 E}$ model are:

$$
\alpha=\frac{\left(-1+\sqrt{1-4 c_{2}\left(1-\frac{1}{\sigma}\right)}\right)}{2 c_{2}}=0 ; \quad \beta=1
$$

Therefore, the velocity and the eddy viscosity approach the shear layer edge linearly. It is interesting to note that the solution for the $k-\varepsilon$ model is also linear for $\sigma_{k}=\sigma_{\varepsilon}=1$ so that the asymptotic behavior of the $k-\varepsilon$ model carries over to the present one-equation model.

The Baldwin-Barth model does not have a solution of the form given by equation 41 . However, as pointed out before, there is no theory available to show that the existence of algebraic solutions is a sufficient, or even a necessary condition to prevent free stream dependency. Numerical test will have to be used to obtain insight into the model characteristics.

Figure 1 shows spreading rates computed with the Baldwin-Barth model as a function of the freestream value, $N_{f}$, and the number of grid points, $n$, across the layer $0 \leq \eta \leq 0.4$. The gridpoints are evenly distributed and the highest freestream values shown in figure 1 are $2 \%$ of the maximum value of $\mathrm{N}$ inside the layer. The Baldwin-Barth model shows a strong sensitivity to the values specified for $N_{f}$, especially as the grid is refined.

Figure 2 shows the computed velocity profiles on the finest grid ( $n=4000$ ) for the highest and the lowest freestream values. It is apparent that the model develops extremely high gradients in the velocity profile for the lower freestream values. The high gradients are the reason why

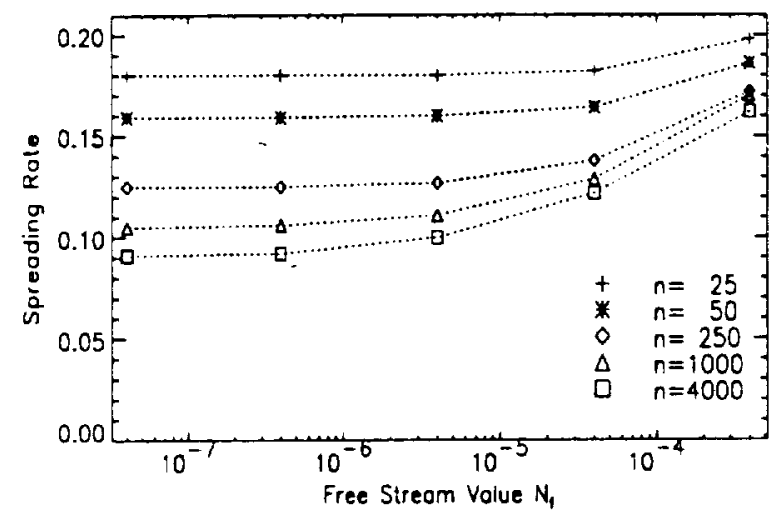

Figure 1. Spreading rates of the Baldwin-Barth model for far wake, depending on freestream values, $N_{f}$, and number of gridpoints. $n$.

the freestream sensitivity does not show up on the coarse grids, where they can simply not be resolved. No grid independent solution could be obtained for the low values of $\mathrm{N}_{\mathrm{f}}$. Even if the number of points is doubled again to $n=8000$, the solution develops even higher slopes and lower spreading rates.

The non-dimensional eddy-viscosities shown in figure 3 demonstrate even more dramatically the changes due to the freestream influence. Like with the $k-\omega$ model, the influence of the freestream values is not confined to the immediate vicinity of the boundary layer edge, but can be observed throughout the layer. In the present case, the maximum of the eddy viscosity changes by about $50 \%$ due to the changes in the freestream value. Note also that the solution for the low freestream values is not grid independent (although 4000 points were used in the computation); even lower values of the eddy viscosity can be achieved by further increasing the number of points. By contrast. the $k-\omega$ model becomes more diffusive with decreasing freestream values and no grid sensitivity is observed.

Spalart and Allmaras (ref. 10) have investigated the behavior of a turbulent front and found that for the Baldwin-Barth model the front propagates into the (physically) incorrect direction away from the non-turbulent region. They also report that their results are dependent on the freestream values specified for the eddy viscosity, consistent with the present findings. Spalar (ref. 20) also reports similar problems with the Baldwin-Barth model as shown in figurel for his computation of a self similar mixing layer. Like in the present calculations, his results are highly sensitive to grid resolution and free stream values. 


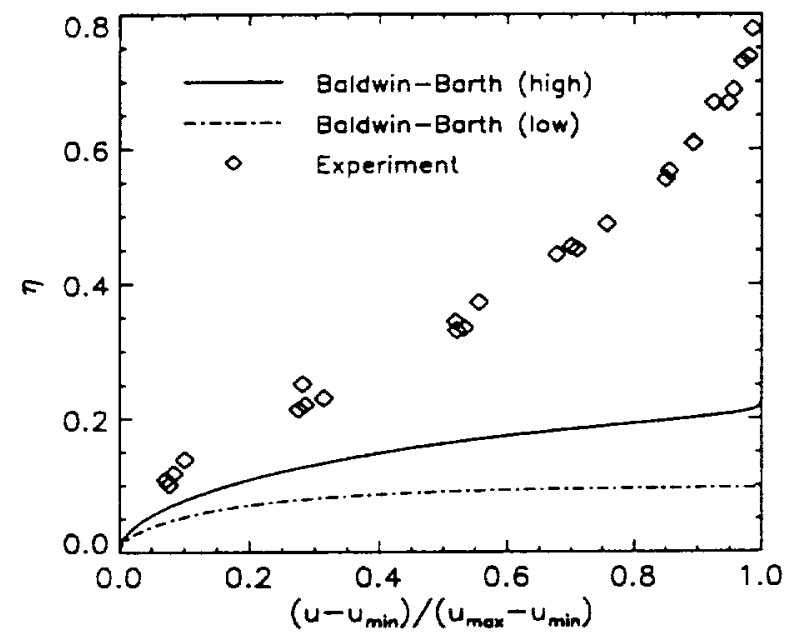

Figure 2. Velocity profiles for far wake with BaldwinBarth model for highest and lowest freestream values (number of gridpoints, $n=4000$.)

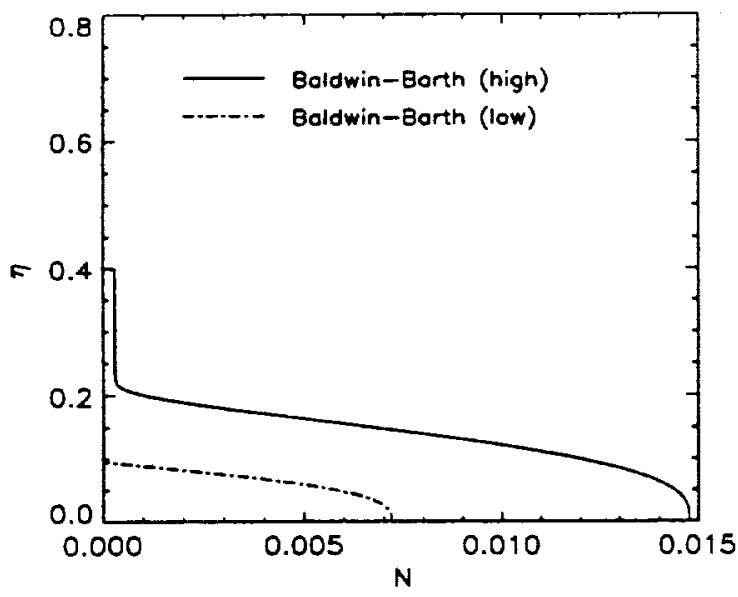

Figure 3. Eddy viscosity profiles for far wake with Baldwin-Barth model for highest and lowest freestream values (number of gridpoints, $n=4000$.)

On the other hand, Baldwin and Barth (ref. 9) have tested the model for a flat plate zero pressure gradient boundary layer (based on a Navier-Stokes code) and found only a moderate dependency on the freestream values. Note however, that Navier-Stokes grids are generally fairly coarse near the boundary layer edge (assuming that all points are plotted in reference 9 , there are about forty points across the boundary layer but only ten of them in the wake region) so that it is very likely that the problem was not resolved in that computation. Note also that the present author has tested the Baldwin-Barth model in NavierStokes codes (ref. 21) and did not realize the severity of the problem for the same reason. However, Rogers (ref. 22) reported that he could not obtain grid independent solutions for airfoil flows with the Baldwin-Barth model using the INS2D Navier-Stokes code.

Goldberg (refs. 23 and 24) has proposed a number of "pointwise" one-equation models, all of which are, in their high Reynolds number version, formally identical to the Baldwin-Barth model (note that the present problems are independent of the low Reynolds number treatment). In reference 23 no problems with the model are reported. In reference 24 problems in the version given in reference 23 are conceded and a new version of the model is proposed. The author claims that the new model avoids the problems near the boundary layer edge due to a regrouping of the terms, but fails to explain why the order of the terms in an equation would have an impact on the solution. In addition, the analysis regarding the behavior of the new model near the edge of the layer is incorrect (only an arbitrary portion of the coefficient in front of the destruction term is considered) and the new model in fact has the same problems as the old one. Again the author does not report any deficiencies with the new model in the testing portion of the paper, although results for self similar shear flows are presented. Furthermore, the results presented for the self similar flows could not be reproduced with any reasonable combination of freestream values and grid distributions by the present author. References 23 and 24 are therefore not considered in the present discussion.

What are the implications of the results shown in Figs. 1-3 for Navier-Stokes applications? There are two different strategies. The first one is to specify small values for the eddy viscosity in the freestream (inflow). The advantage of low values is that they can be specified unambiguouslyvalues that are a fraction of the molecular viscosity will ensure that they are small compared to those inside the layer. However, due to the large gradients developing in this case, no grid independent solutions can be obtained, a situation not acceptable in a Navier-Stokes code. The second strategy is to specify large freestream values (say $x \%$ of the maximum values inside the layer). This in turn puts a large burden on the user, who has to estimate the levels of the eddy viscosity beforehand. Furthermore, the com. putations are sensitive to the value of $x$, so that the results depend on an ambiguous quantity. Note also that computations over complex geometries involve a number of different turbulent layers, and what is a large freestream value for one layer is a small one for an other. Tests have shown 
that in order to reproduce the experimental spreading rates of free shear flows, freestream values of about $20-30 \%$ of the maximum inside the layer have to be used. Values that high would severely impact laminar regions in the flowfield and are certainly not acceptable.

The $(k-\varepsilon)_{1 E}$ model was subjected to the same tests as the Baldwin-Barth model. The model did not show any freestream dependency, as long as the freestream values are small $(<1 \%)$ compared to the values inside the layer. Furthermore the solution for the $(k-\varepsilon)_{1 E}$ model did follow the algebraic solution given by equation 41 near the boundary layer edge. Even for very small values, grid independent solutions were obtained with only about 15 points inside the half-layer.

Table 2 compares the spreading rates of free shear layers as computed with the standard $k-\varepsilon$ and the $(k-\varepsilon)_{1 E}$ model. The experimental values are taken from reference 5 . The Baldwin-Barth model is not included, because no grid- or freestream independent solutions could be obtained. It is interesting to note that the $(k-\varepsilon)_{1 E}$ model gives very similar spreading rates to the standard $k-\varepsilon$ model, despite the fact that Bradshaw's relation, equation 6 , is not generally toue for these flows. The $(k-\varepsilon)_{1 \mathrm{E}}$ model gives somewhat lower spreading rates than the standard $k-\varepsilon$ model for the mixing layer and virtually identical results for the far wake and the plane jet. For the round jet the new model predicts even higher spreading rates than the $k-\varepsilon$ model so that the round jet - plane jet anomaly is somewhat enhanced by the new model.

Table 3. Spreading rates for free shear flows

\begin{tabular}{lccc}
\hline \hline \multicolumn{1}{c}{ Flow } & $\mathrm{k}-\varepsilon$ & $(\mathrm{k}-\varepsilon)_{1 \mathrm{E}}$ & Experiment. \\
\hline Far wake & 0.256 & 0.250 & 0.365 \\
Mixing layer & 0.100 & 0.084 & 0.115 \\
Plane jet & 0.109 & 0.111 & $0.100-0.110$ \\
Round jet & 0.120 & 0.131 & $0.086-0.095$ \\
\hline \hline
\end{tabular}

It is well known that the $k-\varepsilon$ model gives too low spreading rates for the far wake and so does the $(k-\varepsilon)_{1 E}$ model, however, the author agrees with reference 8 that the far wake analysis should not be given the same weight as the other flows, because it is only valid far away from the body. The more important near wake is strongly dependent on how the wake was generated and is not covered by the present analysis.

\section{Equilibrium Boundary Layer Flows}

Wilcox (ref. 5) has popularized the use of defect layer computations for testing turbulence models under equilibrium pressure gradient conditions. The equations describing these flows are identical to the equations for the free shear layers, equations 27 and 28 , with the non-dimensional variables defined as follows:

$$
\begin{aligned}
& u(x, y)=U_{e}-u_{\tau} U(\eta) ; \quad v_{t}(x, y)=N(\eta) U_{e} \delta^{*} \\
& \eta=\frac{y}{\Delta} ; \quad \Delta=\frac{U_{e} \delta^{*}}{u_{\tau}}
\end{aligned}
$$

with $u_{\tau}$ being the friction velocity and $\delta^{*}$ the displacement thickness. The coefficients in equations 27 and 28 are

Table 4. Coefficients for defect layer

\begin{tabular}{ccc}
\hline \hline$S_{U}$ & $S_{N}$ & $V(\eta)$ \\
\hline$\beta_{T}$ & $-\left(1+2 \beta_{T}\right)$ & $-\left(1+\beta_{T}\right) \eta$ \\
\hline \hline
\end{tabular}

with the non-dimensional pressure gradient parameter $\beta_{T}$ defined as:

$$
\beta_{T}=\frac{\delta}{\rho u_{\tau}^{2}} \frac{d p}{d x}
$$

Wall function boundary conditions are specified at the first point off the surface (ref. 5):

$$
\begin{aligned}
& U(\eta)=-\frac{1}{\kappa} \ln (\eta)+U_{0} \\
& N(\eta)=\kappa \eta
\end{aligned}
$$

$U_{0}$ is obtained from the integral constraint:

$\int_{0}^{\infty} U(\eta) d \eta=1$ (for details see (ref. 5)).

At the freestream boundary, $U(\eta)$ is set to zero and $N(\eta)$ to a small freestream value (compared to it's values inside the boundary layer).

As expected, the Baldwin-Barth model has the same freestream dependency as for the free shear layers. Figs. 4 and 5 show computations with this model for the zero pressure gradient boundary layer experiment of Wieghardt (ref. 4) on two different grids and with two different freestream values. The coarse grid consists of 70 points 


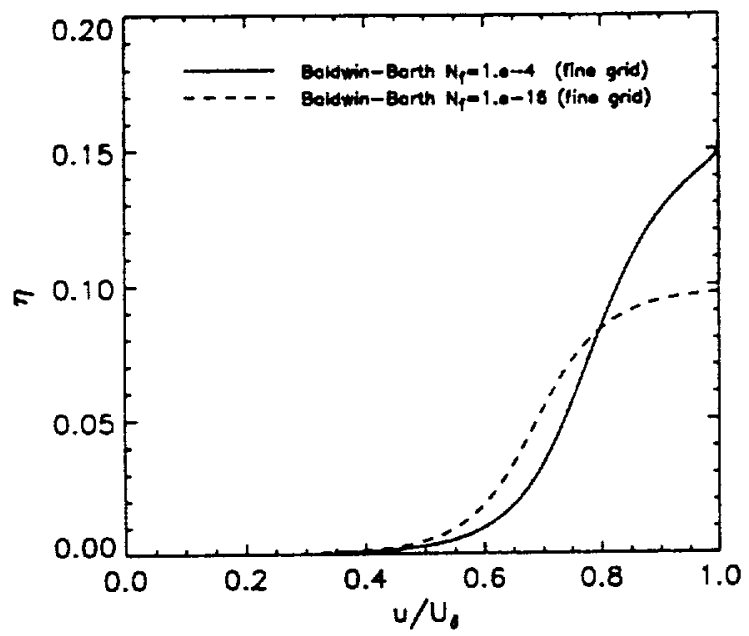

Figure 4 . Velocity profiles for defect layer with Baldwin-Barth model and two different freestream values, $N_{f}$, (fine grid with $n=1000$ ).

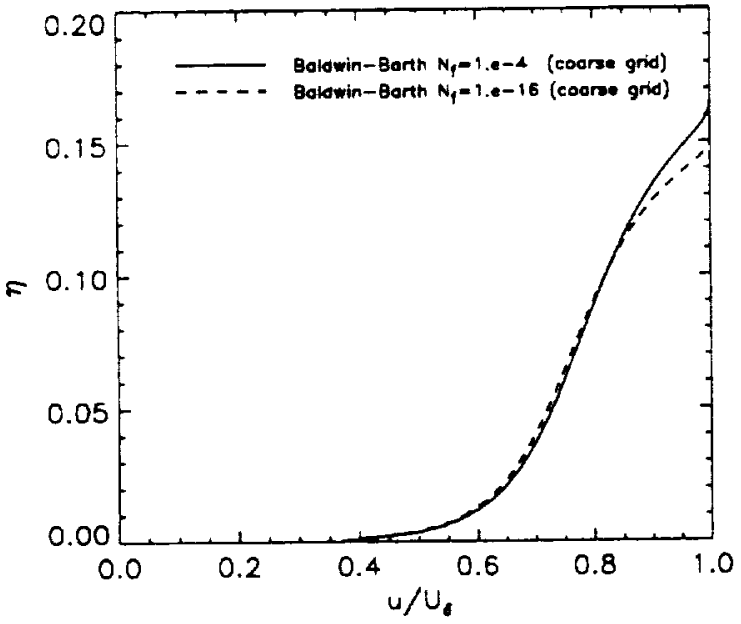

Figure 5. Velocity profiles for defect layer with Baldwin-Barth model and two different freestream values, $N_{f}$, (coarse grid with $n=70$ ).

and the fine grid of 1000 points; the high freestream value is about $1 \%$ of the maximum eddy viscosity value inside the boundary layer. Again the solution develops extreme gradients near the boundary layer edge for the fine grid and the low freestream values. Note that only a moderate sensitivity can be observed on the coarse grid. Note also that 70 points in the defect part of the boundary layer is a very fine grid in Navier-Stokes computations. It is obvious that the freestream sensitivity could not be resolved in (ref. 9). As in the free shear layer computations, the influence of the freestream values and grid densities is not confined to the vicinity of the boundary layer edge, but affects the whole layer. Table 5 shows the computed $c_{p}$-values for the different cases, reemphasizing this point.

Table 5. $\mathrm{C}_{\mathrm{f}}$ results for the Baldwin-Barth model for different grids and freestream values, $N_{f}$

\begin{tabular}{cccc}
\hline \hline Gridpoints & High $\mathrm{N}_{\mathrm{f}}$ & Low $_{\mathrm{f}}$ & Experiment \\
\hline $\mathrm{n}=70$ & 0.0023 & 0.0022 & 0.0025 \\
$\mathrm{n}=1000$ & 0.0021 & 0.0018 & 0.0025 \\
\hline
\end{tabular}

No grid converged solution could be obtained with the Baldwin-Barth model for the low freestream value. From the present study it is not clear, whether an asymptotic solution exists for this model as $\mathrm{N}_{\mathrm{f}}$ goes to zero and the number of grid points goes to infinity. Whenever the number of grid-points was increased, the solution changed with an increase in the slope near the edge and a lower eddy viscosity inside the layer.

Figure 6 shows a comparison of results for the zero pressure gradient case of Wieghardt (ref. 4) for the $k-\varepsilon$ and the $(k-\varepsilon)_{1 E}$ models. Both models give very accurate velocity

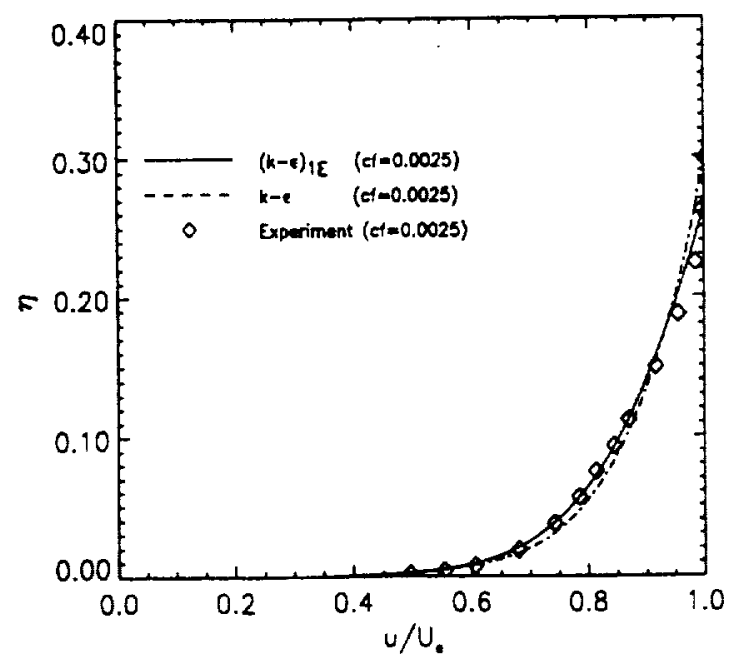

Figure 6. Velocity profiles for defect layer, $\beta_{\mathrm{T}}=0$. 
profiles and $c_{f}$ predictions.

Figure 7 shows results for the adverse pressure gradient flow of Clauser (ref. 4) for a non-dimensional pressure gradient of $\beta_{T}=8.7$. It is well known that the standard

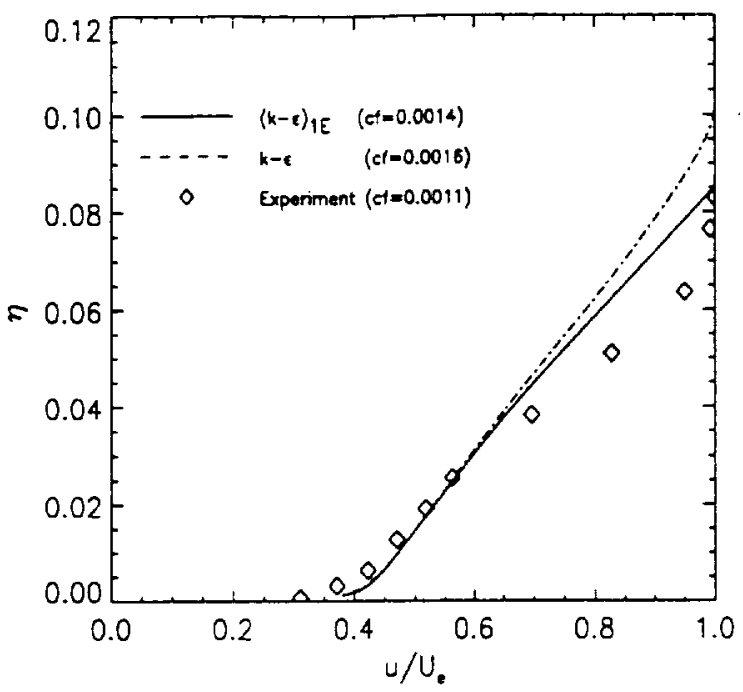

Figure 7. Velocity profiles for defect layer, $\beta_{\mathrm{T}}=8.7$.

$k-\varepsilon$ model overpredicts the skin friction for adverse pressure gradient flows, in this case by about $50 \%$. Note that the apparent differences in boundary layer thickness between the computations and the experiment are a result of the definition of $\eta$, involving the friction velocity $u_{\tau}$. The introduction of Bradshaw's relation, equation 6 , into the $(k-\varepsilon)_{1 E}$ model obviously improves the predictions, but the skin friction is still too high by about $30 \%$. Due to the close relationship of the $(k-\varepsilon)_{1 E}$ model to the standard $k-\varepsilon$ model, it had to be expected that the deficiency in the adverse pressure gradient behavior of the k- $\varepsilon$ model would not entirely be avoided by the new model.

\section{Navier-Stokes Computations}

All of the following incompressible test cases have been computed with the NASA Ames INS2D and INS3D codes (ref. 25); the compressible flow was solved with the NASA Langley CFL3D code (ref. 26). All flows in this study are part of a test base assembled by the author to evaluate the performance of turbulence models (refs. 11 14). The flows have been set up in a way to match the experimental boundary conditions as closely as possible. Furthermore, all computations are performed on grids that have been shown to produce grid independent solutions.

It was initially intended to compare results of all three models for the following Navier-Stokes applications. However, due to the severe deficiencies discovered in the Baldwin-Barth model for the equilibrium flows, the model was dropped from the study. It is felt that results obtained with that model bear little meaning, since they are invariably either grid- or freestream dependent.

\section{Flat Plate Zero Pressure Boundary Layer}

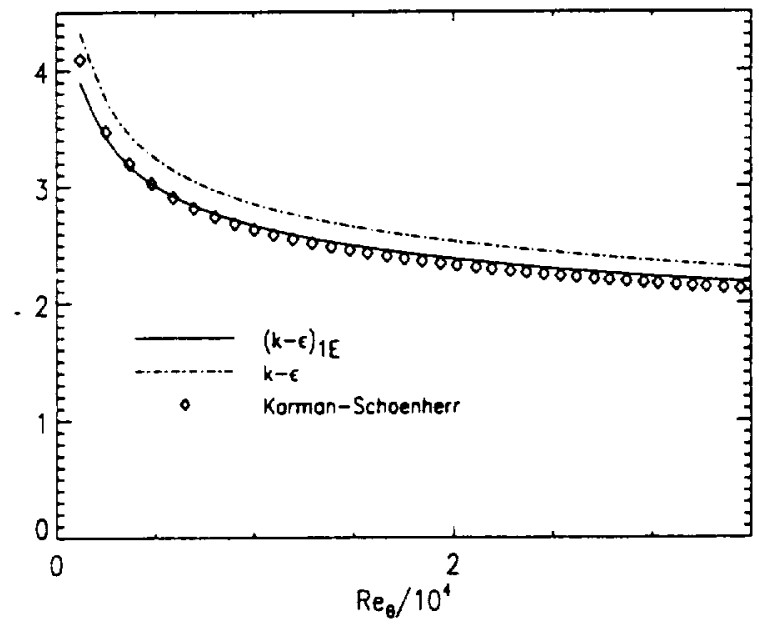

Figure 8. Skin-friction coefficient for flat plate boundary layer.

Figure 8 shows a comparison of the computed wall skin friction coefficients, $c_{f}$, versus displacement thickness, $\theta$, for a flat plate zero pressure gradient boundary layer. The computations are compared with the experimental correlation of v. Karman and Schoenherr. The low Reynolds number version of the $k-\varepsilon$ model is due to Launder and Sharma. The $(k-\varepsilon)_{1 E}$ model is in good agreement with the experiments, whereas the Launder-Sharma model gives values about $10 \%$ too high. The new model gives accurate results, as long as the first grid point satisfies $\mathrm{y}_{1}{ }^{+}<\sim 2.5$.

The velocity profiles in inner coordinates are depicted in figure 9. Again the $(k-\varepsilon)_{1 E}$ model is in better agreement with the law of the wall than the Launder-Sharma model. The good results of the one-equation model are not surprising since the low Reynolds number functions have been optimized for this flow. Note that the discrepancies between the computations of the $k-\varepsilon$ model and the 


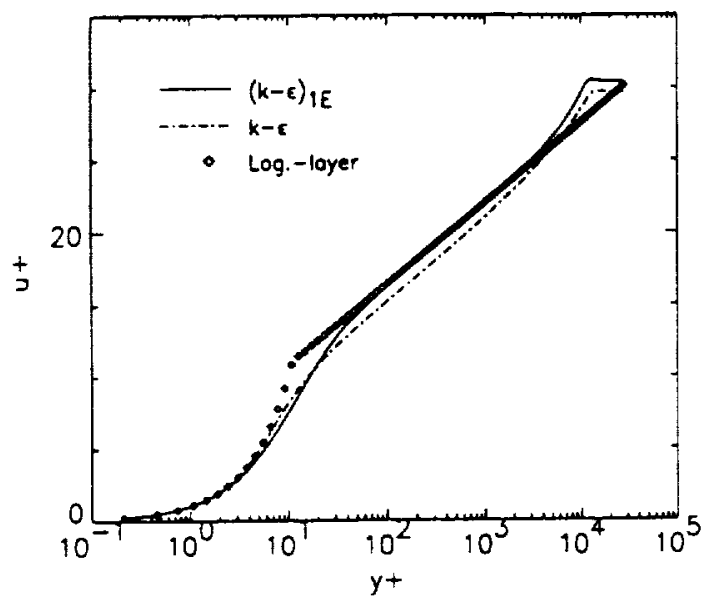

Figure 9. Velocity profiles for flat plate boundary layer in inner coordinates

experimental correlations are a result of the low Reynolds number terms in the Launder-Sharma model. The high Reynolds number version of the model with wall functions gives the correct skin friction as demonstrated by the equilibrium computations shown in figure 6. Results similar to the present ones have been reported by Coakley (ref. 27) for the Launder-Sharma model.

\section{Samuel-Joubert Adverse Pressure Gradient Flow}

The Samuel-Joubert flow (ref. 28) is one of the standard test cases for non-equilibrium adverse pressure gradient flows. In this flow, a flat plate boundary layer develops under an increasingly adverse pressure gradient. The unit Reynolds number is $1.7 \times 10^{6}$. The flow is retarded, but not separated. The computations have been performed on a $90 \times 90$ (verified on a $120 \times 120$ ) grid.

Figure 10 shows a comparison of the computed and the experimental wall shear stress distributions. The standard $k-\varepsilon$ model gives higher $c_{f}$-vaiues than the experiments already close to the inflow as a result of its failure to accurately predict a zero pressure gradient boundary layer (fig. 8). The $(k-\varepsilon)_{1 \mathrm{E}}$ model is in better agreement with the data, but develops somewhat too high wall-shear levels as the pressure gradient becomes more severe. This is in agreement with the findings for the equilibrium flows.

Since the wall shear depends on the specifics of the damping functions, a better comparison of the high Reynolds

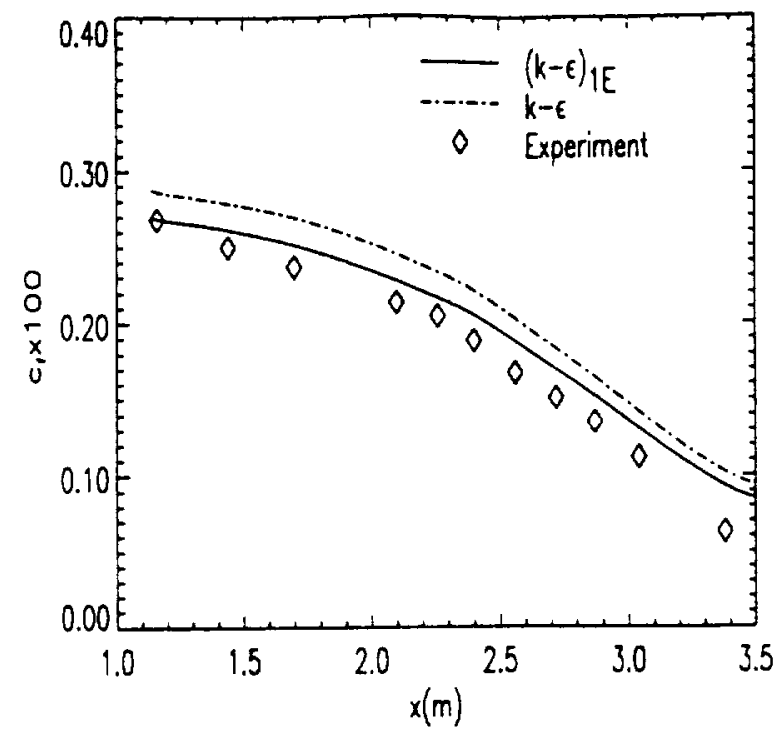

Figure 10. Skin-friction for Samuel-Joubert flow.

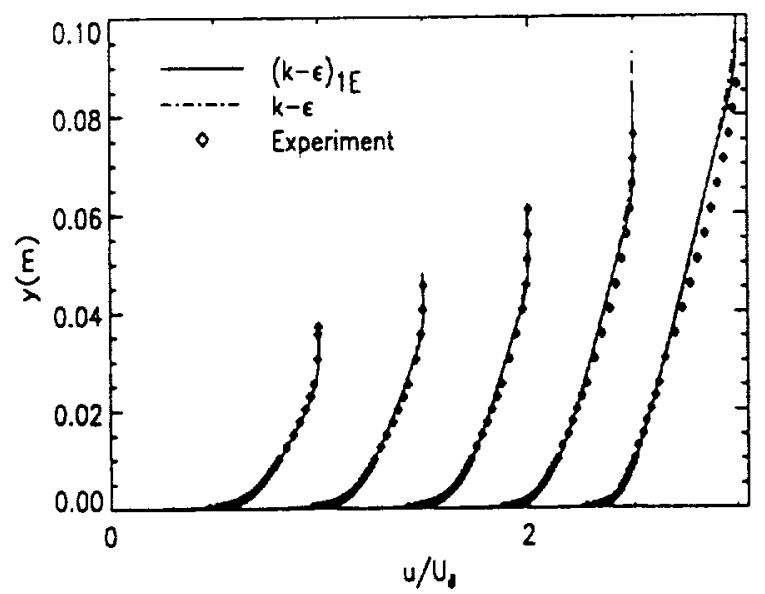

Figure 11. Velocity profiles for Samuel-Joubert flow at $\mathrm{x}=1.16,1.76,2.26,2.87,3.40 \mathrm{~m}$.

number behavior of the models can be obtained from the velocity profiles. Figure 11 shows that the two models produce almost identical results - a testimony to the correctness of the assumptions leading to the $(k-\varepsilon)_{1 E}$ model for this flow. It was found previousiy that the Samuel-Joubert flow is not a very severe test case and non-equilibrium effects are small, so that a good correspondence between the two models had to be expected. 


\section{Driver Separated Adverse Pressure Gradient Flow}

In Driver's flow (ref. 29), a turbulent boundary layer develops in the axial direction of a circular cylinder. A strong adverse pressure gradient is imposed on the flow by diverging wind tunnel walls plus suction applied at these walls. The pressure gradient is strong enough to cause the flowfield to separate. The inflow Reynolds number is $2.8 \times 10^{5}$ based on the diameter, $D$, of the cylinder. The inflow boundary layer thickness is about $0.2 \mathrm{D}$. The experiments offer independent wall-skin friction measurements and it was found in previous tests (ref. 12) that the data are highly self-consistent and well suited to test models under strong pressure gradient conditions. The computations are performed on a $60 \times 3 \times 60$ (verified on a $90 \times 3 \times 90$ ) grid.

Figure 12 shows the wall skin friction coefficient for this flow. As in previous comparisons, the standard $\mathrm{k}-\varepsilon$ model

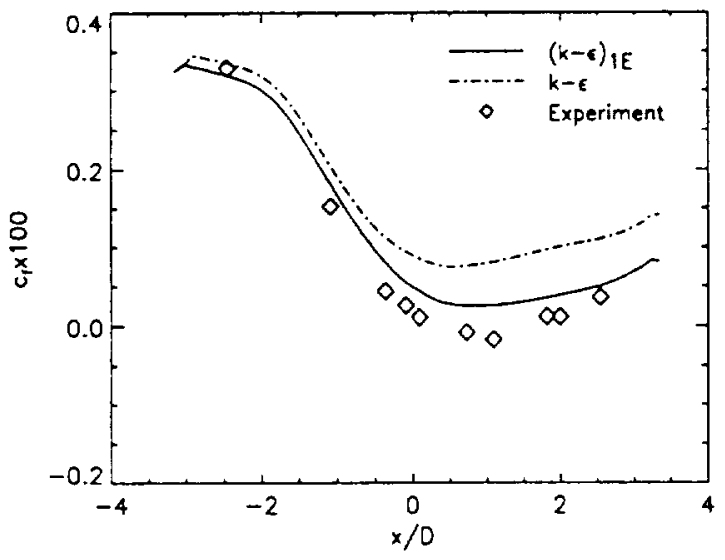

Figure 12. Skin-friction for Driver's case CS0.

predicts significantly higher values than the experiment, again partly due to the low Reynolds number form of the equations. The $(k-\varepsilon)_{1 E}$ model is in significantly better agreement with the data, but still somewhat too high, especially in the region where the experiment shows separation.

The wall pressure distribution shown in figure 13 is a better indicator of the high Reynolds number performance of the models. The one-equation model is a little closer to the data, but both models underpredict the viscous-inviscid interaction. This can also be inferred from figure 14, showing the velocity profiles. Again it can be seen that the $(k-\varepsilon)_{1 E}$ model predicts a stronger retardation due to the pressure gradient, but not enough to be in good agreement

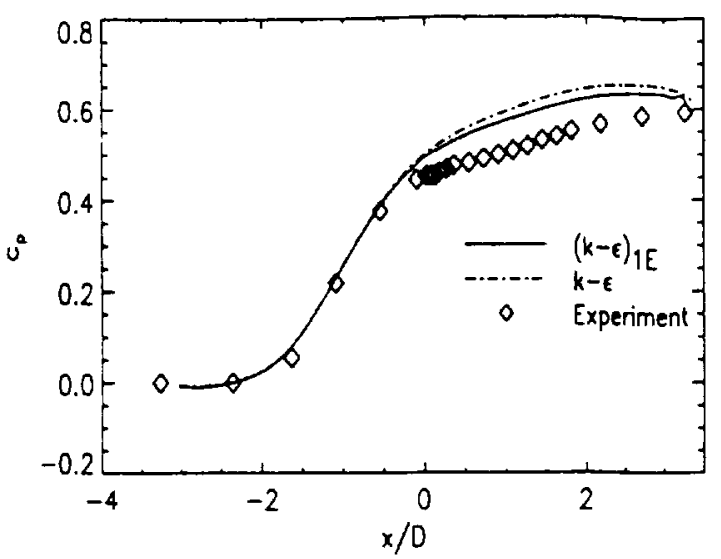

Figure 13. Pressure distribution for Driver's case CSO.

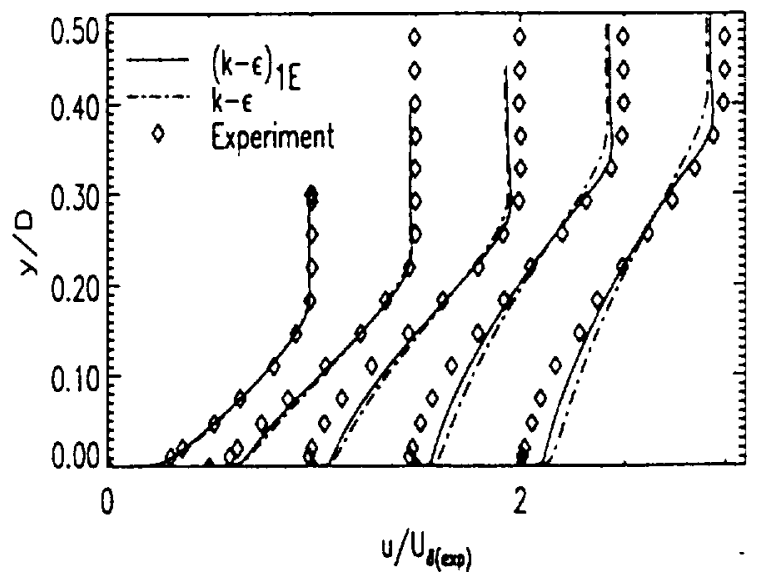

Figure 14. Velocity profiles for Driver's case CSO at $x /$ $\mathrm{D}=-0.544,-0.091,0.363,1.088,1.633$.

with the data. The reason for the failure to predict the retardation of the velocity profiles lies in an overprediction of the turbulent shear stress, as can be seen in figure 15 . The experimental data are shown in a cartesian system aligned with the surface of the cylinder and in a streamline coordinate system aligned with the streamline in the middle of the boundary layer $\left(u / u_{\max }=0.5\right)$. It was pointed out recently (ref. 30 ) that a streamline coordinate system is the most meaningful system to compare turbulent shear stresses; a cartesian system can lead to incorrect conclusions if the streamlines of the flow are not parallel to the surface. In previous computations, it was found very 


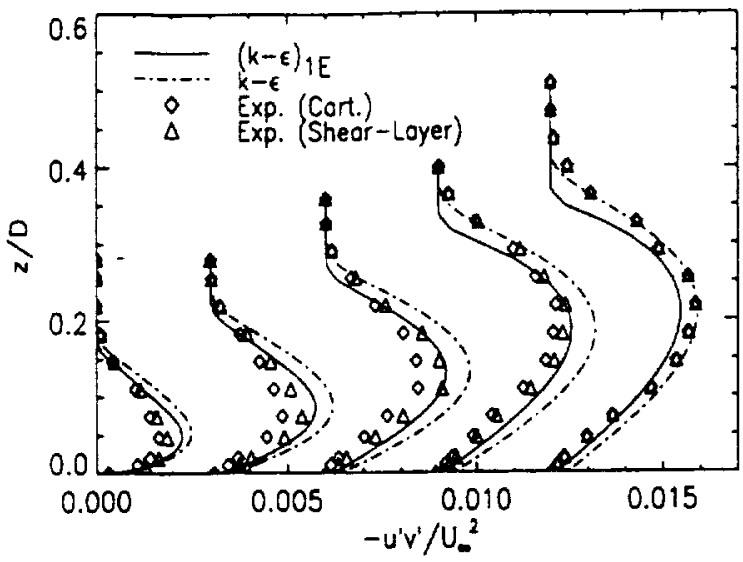

Figure 15. Shear stress profiles for Driver's case CSO at $x / \mathrm{D}=-0.544,-0.091,0.363,1.088,1.633$.

important to accurately compute the turbulent shear stresses ahead of the separation (first two profiles in fig. 15) in order to correctly predict this flow. Both models give too high values in that region. Note however that the $(k-\varepsilon)_{1 E}$ model is in better agreement with the data than the two-equation model. As the flow encounters more severe non-equilibrium conditions, the differences between Bradshaw's relation, equation 6 , and the relation enforced by the two-equation model, equation 7 , become more severe and the predictions of the two models start to deviate. Figure 16 shows the ratio of production versus dissipation as predicted by the standard $\mathbf{k}-\varepsilon$ model at the location of the maximum turbulent shear stress. This ratio is an indicator of the non-equilibrium effects and enters into equation 7. Since Bradshaw's relation is generally more realistic than equation 7 , the $(k-\varepsilon)_{1 E}$ model gives better results than the standard k- $\varepsilon$ model.

\section{Backward Facing Step Flow}

The backward facing step is one of the most widely used test cases for turbulence model evaluation. While early results for this flow indicated that the $k-\varepsilon$ model underpredicts the reattachment length by $\sim 30 \%$, more recent results have shown that the model is off by only about $5 \%$. The earlier computations had not enough resolution to accurately predict the flow. The test case in this study is the flow of Driver and Seegmiller (ref. 31). The Reynolds number, based on the upstream momentum thickness $\theta$ is $R e_{\Theta}=5,000$ and the ratio of the boundary layer thickness to step height is about 1.5. The expansion ratio is 1.125. The computations have been performed on a

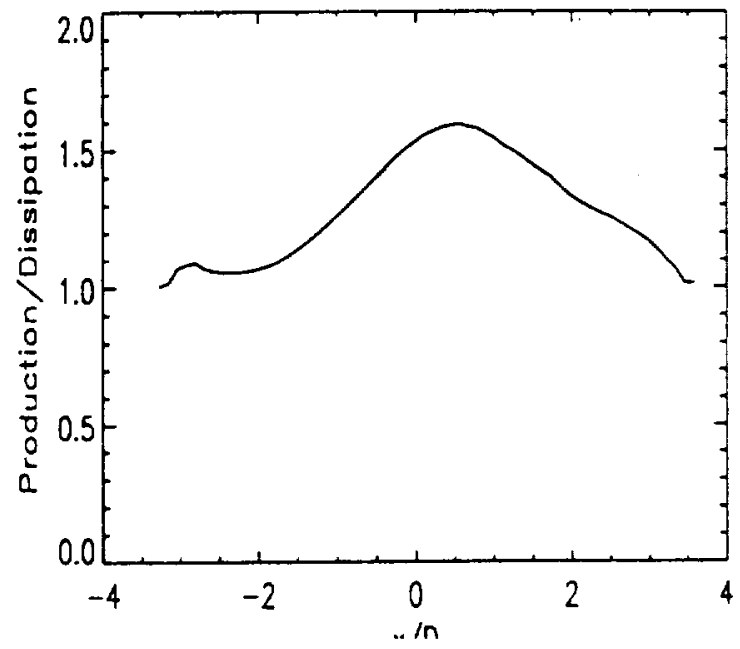

Figure 16. Ratio of Production/Dissipation for Driver's case CSO as computed from the $k-\varepsilon$ model.

$120 \times 120$ grid with substantial grid refinement near the step. The computations are virtually identical to those performed on a $240 \times 240$ grid.

Figure 17 shows the computed and the experimental skin friction distributions. The standard $k-\varepsilon$ model underpre-

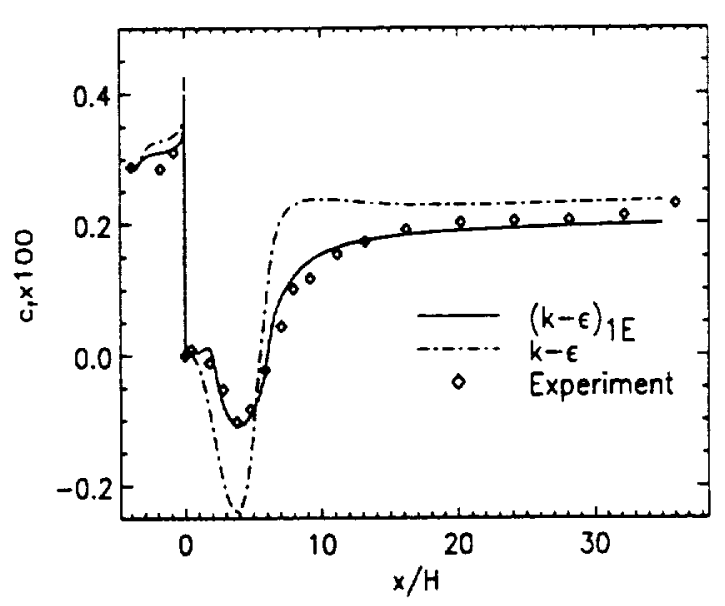

Figure 17. Skin-friction for backward facing step flow.

dicts the reattachment location by about $5 \%$ and is generally not in good agreement with the data in the separated region and near reattachment. Different low Reynolds number forms of this model give different skin friction 
distribution, so that this behavior is certainly a result of the low-Reynolds number terms. The $(k-\varepsilon)_{1 E}$ model is in very good agreement with the data. The reattachment location is predicted within experimental uncertainty, and there are no overshoots near reattachment. Especially impressive is the skin friction recovery downstream of reattachment, where other models tend to fall more severely below the experiments (refs. 11, 12, and 14).

The velocity profiles depicted in figure 18 show that the high Reynolds number differences between the models are amazingly small. The velocity profiles are almost identical

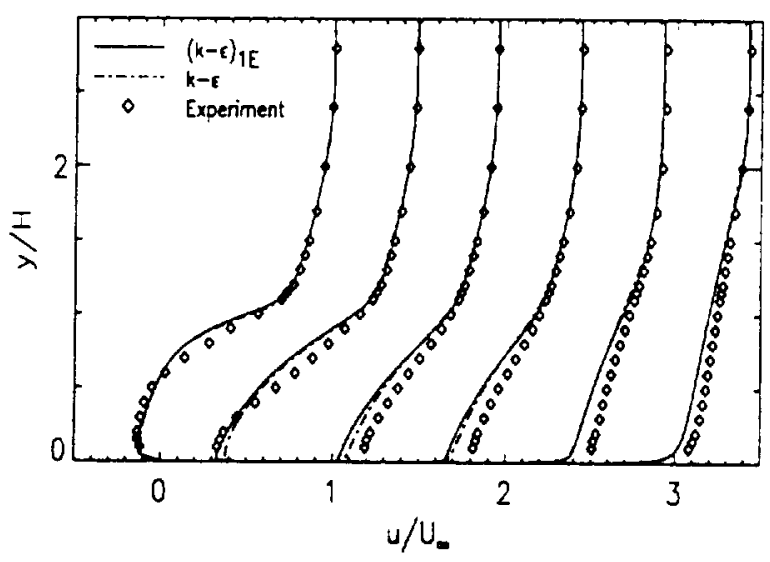

Figure 18. Velocity profiles for backward facing step flow at $x / h=2,4,6.5,8,14,32$.

even inside the separation bubble and it appears again that the two-equation model does not offer an advantage over the one-equation model. Both of the present models fail to predict the recovery of the velocity profiles downstream of reattachment. This is a general problem with existing models and has been observed before (refs. 11, 12, and 14).

Turbulent shear stress profiles are shown in figure 19. Again, the experimental profiles are depicted in a cartesian and in a shear layer coordinate system. The shear layer direction is defined as the direction of the streamline at the location where the velocity in the profile is half the difference between the minimum and the maximum velocity. The results of the two models are very similar. The $(k-\varepsilon)_{1 E}$ model predicts slightly lower shear stress leveis due to the adverse pressure gradient, again as a results of the introduction of Bradshaw's relation, equation 6. It is for this reason that the reattachment location is predicted more accurately by that model. Both models predict the location

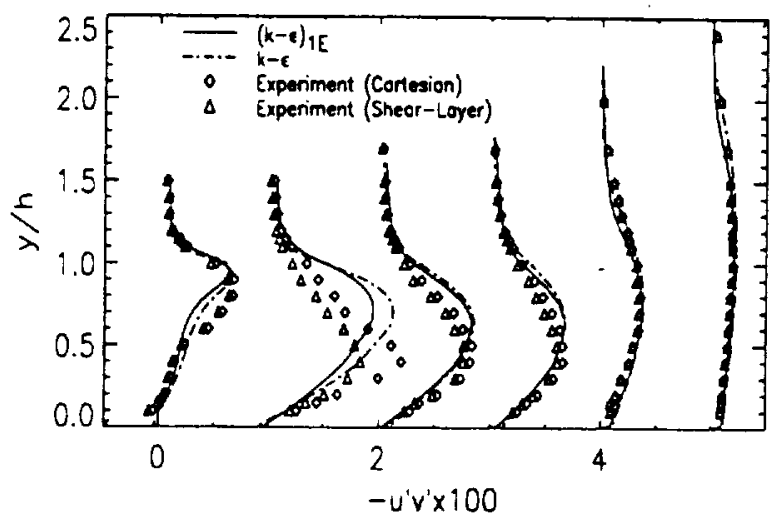

Figure 19. Shear stress profiles for backward facing step flow at $x / h=2,4,6.5,8,14,32$.

of the maximum shear stress in the recirculation region at a different location than given by the experiment - a shortcoming also observed with other models. This might be party responsible for the failure of the models to predict the correct flow recovery further downstream.

\section{NACA 4412 Airfoil Flow}

The following flowfield was investigated experimentally by Coles and Wadcock (ref. 32). It is a NACA 4412 airfoil close to maximum angle of attack at low speed. The chord Reynolds number is $\operatorname{Re}_{c}=1.52 \times 10^{6}$ and the angle of attack is $\alpha=13.87^{\circ}$. In the experiment the transition was fixed at $x / c=0.023$ and 0.1 on the upper and lower surface respectively. As reported in (ref. 33), a laminar separation bubble appears in the calculations on the upper surface, in front of the transition point. Laminar separation is not observed in the experiments, indicating that transition may take place before the trip is reached. The computed results are insensitive to the transition location as long as it is upstream of the laminar separation point. For the following results the transition location was close to the leading edge, as predicted by the models.

A novelty in the present computations, compared to earlier ones (refs. 12 and 33) is that the wind tunnel walls are included in the predictions. The computations were performed on two grids, a $101 \times 101 \mathrm{H}$-grid for the wind tunnel and a $321 \times 51 \mathrm{C}$-grid for the airfoil, linked by the chimera capability of the INS2D code. The solid walls of the wind tunnel are simulated as slip surfaces. The experimental data were corrected for the fact that the reference pressure $p_{r}$ in the experiments was not equal to $p_{\infty}$ due to 
the pressure tab having been close to the airfoil and therefore not in the undisturbed flow regime. The difference between the two pressures is obtained by comparing $p_{r}$ and $p_{\infty}{ }^{\prime}$ as computed by the code at the location of the pressure tab. The difference is largely independent of the turbulence model employed. The resulting corrections are $p_{\infty} \equiv p_{r}(1-0.06)$ for the pressure and $U_{\infty} \equiv U_{r}(1+0.06)$ for the velocity.

Figure 20 shows a comparison of computed and experimental velocity profiles. The results produced by the

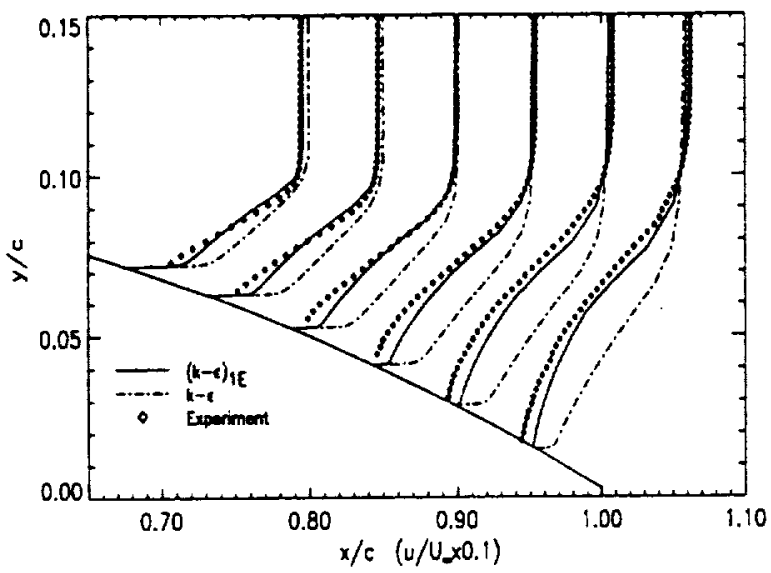

Figure 20. Velocity profiles for NACA 4412 airfoil, $\alpha=13.87, \operatorname{Re}=1.7 \times 10^{6}$.

one-equation model are better than those of the twoequation model for the same reasons as in the previous flows. Differences between the models are larger than for Drivers's case, indicating that the flow is even more out of equilibrium.

A significant improvement with the data is achieved compared to computations without tunnel walls (refs. 12 and 33), especially with regard to the boundary layer thicknesses (which were significantly underpredicted before) and the velocities outside the boundary layer. However, figure 21 shows that the experimental pressure distribution still could not be reproduced accurately. The computed suction peak is higher than in the experiment, resulting in a more severe pressure gradient along the upper surface. The reason for the discrepancies is not entirely clear, but is not a result of deficiencies in the turbulence models, since models which almost duplicate the velocity profiles still don't match the wall pressure. Note however, that in the experiment a rectangular test section was mounted inside a round wind tunnel, with the walls of

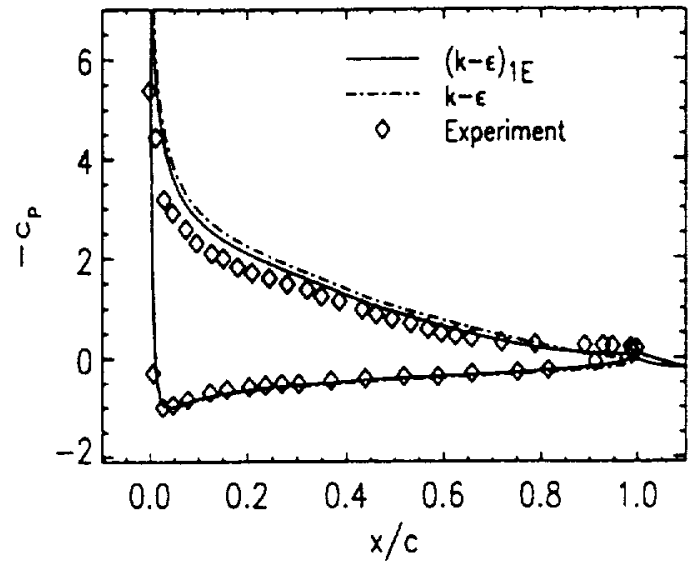

Figure 21. Pressure distribution for NACA 4412 airfoil, $\alpha=13.87, \operatorname{Re}=1.7 \times 10$.

the rectangular section extending only to about half a chord length upstream of the leading edge of the airfoil. The combined blockage effect of the airfoil and of the inner test section could have interfered with the flow ahead of the airfoil. Since the experimental inflow conditions into the test section are not known and since uniform flow so close to the airfoil is unrealistic, the wind tunnel walls simulated in the computations were extended to five chords upstream of the leading edge and uniform flow was specified. These differences in geometry can very well be responsible for the discrepancies in the pressure distributions.

It should however be noted, that the differences in the pressure distribution are not responsible for the lack of separation in the models, since the computed pressure rise is actually higher than the one in the experiments.

\section{Transonic Bump Flow}

The final test case is the transonic bump flow of Bachalo and Johnson (ref. 34). In this experiment an axisymmetric boundary layer interacts with a shock wave created by a circular arc. Only the highest Mach number case $(\mathrm{Ma}=0.925)$ will be shown. The number of gridpoints was $150 \times 3 \times 80$ which was found to produce grid independent results in a previous study (ref. 13).

Figure 22 shows the wall pressure distribution computed by the two models, compared with the experiments. The $(k-\varepsilon)_{1 E}$ model gives significantly better results than the 


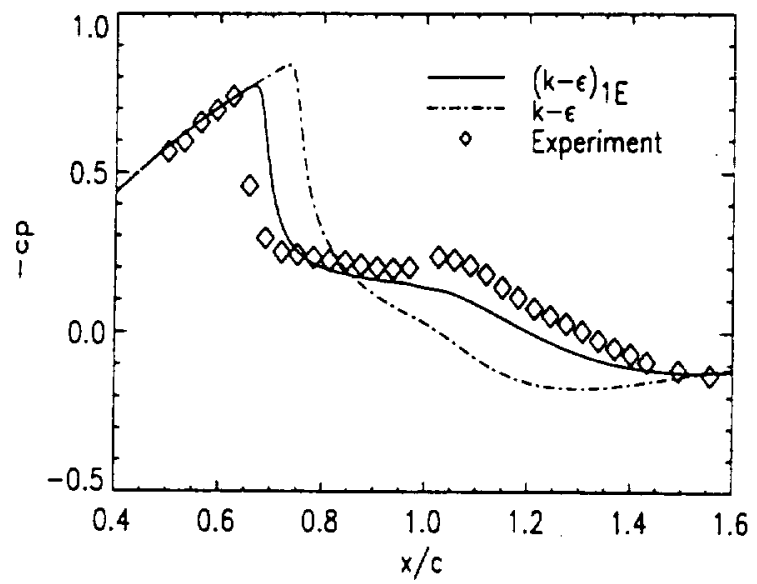

Figure 22. Pressure distribution for transonic bump flow, $\mathrm{M}=0.925$.

standard $k-\varepsilon$ model. Note that this flow is the strongest non-equilibrium flow in the study and the differences between equations 6 and 7 are therefore largest. Again, Bradshaw's relation leads to a significant improvement in the comparison, although the shock is still somewhat too far downstream with the new model.

\section{Conclusions}

The connection between one- and two-equation models of turbulence has been reexamined. It was found that the standard $\mathrm{k}-\varepsilon$ model can be transformed into a one-equation model based on only two assumptions. The first one is that the diffusion coefficients in the $k$ - and the $\varepsilon$-equations are the same. By enforcing this condition in the $\mathrm{k}-\varepsilon$ model, it was shown that only minor changes resulted from it. The second assumption is Bradshaw's relation that the turbulent shear stress is proportional to the turbulent kinetic energy. This assumption corresponds to Production $_{k}=$ Dissipation $_{k}$ in standard two equation models and is therefore exact for equilibrium flows. For non-equilibrium flows, Bradshaw's relation is actually better confirmed by experiments than the relation enforced by the standard $k-\varepsilon$ model. The new model was termed $(k-\varepsilon)_{1 E}$ model and tested against the Baldwin-Barth model and the standard $k-\varepsilon$ model.

Free-shear layer computations have shown that the Baldwin-Barth model is ill-conditioned near the boundary layer edge. The model does not posses an algebraic solution in that region and produces unlimited gradients in the velocity as the grid is refined. Furthermore, results are sensitive to the freestream values specified outside the layer. No grid and freestream independent results could be obtained with this model and it was for this reason not included in the rest of the study. The reason for the failure of the model lies in a destruction term that does not follow from the transformation of the two-equation model, but was introduced on dimensional arguments.

The findings for the Baldwin-Barth model re-emphasize that the behavior of turbulence models near the turbulentnon-turbulent interface is one of the most important aspects of turbulence modeling. Shortcomings in that area are not confined to the immediate vicinity of the interface, but change the solution through the whole layer, essentially nullifying the calibration process. Unfortunately, not enough attention is paid to this problem in the derivation and calibration of most new models, leaving the door open for potentially devastating failures. This is also true for Reynolds stress models.

The new one-equation model does not suffer from these deficiencies and shows a very close similarity to it's parent two-equation model near the boundary layer edge. A comparison of the free shear layer results has shown that the predictions of the one- and two-equation models are fairly close to one another. Both models predict virtually identi$\mathrm{cal}$ results for the far wake and the plane jet. Compared to the $\mathrm{k}-\varepsilon$ model, the new model predicts about $15 \%$ lower spreading rates for the plane mixing layer and about $10 \%$ higher ones for the round jet.

For zero pressure gradient boundary layers, the new model gives almost identical results to the $k-\varepsilon$ model, except for some deficiencies in the low Reynolds number behavior of the two-equation model. A number of increasingly stronger adverse pressure gradient flows has shown that the results of the new model improve compared to the $k-\varepsilon$ model predictions as the non-equilibrium effects become more important. The improved results confirm that the main assumption going into the new model is more realistic than the relation enforced by the $k-\varepsilon$ model.

The computations have shown that there is little advantage in using a two- over a one-equation model in predicting turbulent shear flows. The new one-equation model has produced better results than the two-equation model for all but the free mixing layer and the round jet. However, due to the close relationship to the standard $k-\varepsilon$ model, the new model still underpredicts the retardation of flows under adverse pressure gradient conditions.

Appendix: Transforming the $k-\omega$ Model 
The transformation leading to the $(k-\varepsilon)_{1 \mathrm{E}}$ can be used to transform any two-equation model into a one-equation model. An example is the $k-\omega$ model of Wilcox (ref. 5):

$$
\begin{aligned}
& \frac{D k}{D t}=v_{t}\left(\frac{\partial u}{\partial y}\right)^{2}-\beta^{\circ} k \omega+\frac{\partial}{\partial y}\left(\sigma v_{t} \frac{\partial}{\partial y}(k)\right) \\
& \frac{D \omega}{D t}=\alpha\left(\frac{\partial u}{\partial y}\right)^{2}-\beta \omega^{2}+\frac{\partial}{\partial y}\left(\sigma v_{t} \frac{\partial}{\partial y}(\omega)\right)
\end{aligned}
$$

In the $\mathrm{k}-\omega$ model the definition of the eddy viscosity is:

$$
v_{i}=\frac{k}{\omega}
$$

With the help of equations 3 and 6 the following one-equation model can be derived (high Reynolds number form):

$$
\frac{D \tilde{v}_{t}}{D t}=c_{1} \bar{v}_{t}\left|\frac{\partial u}{\partial y}\right|+c_{2} \bar{v}_{t} \frac{\frac{\partial}{\partial y}\left|\frac{\partial u}{\partial y}\right|}{\left|\frac{\partial u}{\partial y}\right|} \frac{\partial \bar{v}_{t}}{\partial y}+\frac{\partial}{\partial y}\left(\sigma \bar{v}_{t} \frac{\partial}{\partial y}\left(\bar{v}_{s}\right)\right)
$$

The constants in this model follow directly from the $k-\omega$ constants:

$$
c_{1}=\frac{\beta}{a_{1}}-a_{1} \alpha=0.0833 ; \quad c_{2}=2 \sigma=1
$$

with:

$$
\alpha=\frac{5}{9} ; \quad \beta=0.075 ; \quad \sigma=0.5 ; \quad \beta^{\circ}=a_{1}^{2}=0.09
$$

Note that the diffusion coefficients in the $k$ - and the w-equation are equal so that no terms proportional to the difference of these two constants appears (see eq. 8).

First tests with this model have shown that the solutions develope large gradients in the velocities near the boundary layer edge, similar to the Baldwin-Barth model. This is possibly due to the small diffusion coefficient of $\sigma=0.5$. Note also that the $k-\omega$ model has a strong dependency on freestream values (ref. 17), which seems to carry over to the one-equation model. More analysis and careful testing, as well as a possible recalibration of the coefficients will be required before the model can be applied to engineering flows.

\section{References}

1. Baldwin, B. S.; and Lomax, H.: Thin Layer Approximation and Algebraic Model for Separated Turbulent Flows. AIAA Paper 78-0257, Huntsville, Ala, 1978.

2. Johnson, D. A.; and King, L. S.: A Mathematically Simple Closure Model for Attached and Separated Turbulent Boundary Layers. AIAA J., vol. 23, Nov. 1985, pp. 1684-1692.

3. Jones, W. P.; and Launder B. E.: The Calculation of Low-Reynolds-Number-Phenomena with a TwoEquation Model of Turbulence. Int. J. Heat Mass Transf., vol. 16, 1973, pp. 1119-1130.

4. Kline, S. J.; Cantwell, B. J.; Lilley, G. M., eds.: 19801981 AFOSR-HTTM Stanford Conference on Complex Turbulent Flows. Comparison of Computation and Experiment, Stanford University, Stanford, Calif., 1981.

5. Wilcox, D. C.: Turbulence Modeling for CFD. Publisher: DCW Industries, Inc., La Canada, Calif., 1993.

6. Bradshaw, P.; Ferriss, D. H.; and Atwell, N. P.: Calculation of Boundary Layer Development Using the Turbulent Energy Equation. J. Fluid Mechanics, vol. 23, pp. 31-64.

7. Nee, V. W.; and Kovasznay, L.S.G.: Simple Phenomenological Theory of Turbulent Shear Flows. The Physics of Fluids, vol 12, no.3, March 1969. pp. 473-484.

8. Gulyaev, A. N.; Kozlov, V. Y.; and Secundov, A. N.: Universal Turbulence Model “ $v_{t}-92$ ". Ecolen Repont, Moscow, 1993.

9. Baldwin, B. S.; and Barth, T. J.: A One-Equation Turbulence Model for High Reynolds Number Wall Bounded Flows. NASA TM-102847, 1990.

10. Spalart, P. R.; and Allmaras, S. R: A One-Equation Turbulence Model for Aerodynamic Flows, La Recherche Aerospatiale, no. 1, 1994, pp. 5-21.

11. Menter, F. R.: A Critical Evaluation of Promising Eddy-Viscosity Turbulence Models. Proceedings Intern. Symp. on Turbulence, Heat and Mass Transfer, Portugal, 1994, pp. 13.4.1-13.4.6. 
12. Menter, F. R.: Zonal Two Equation k-w Turbulence Models for Aerodynamic Flows. ALAA Paper 932906, Orlando, Fla. 1993.

13. Menter, F. R.; and Rumsey, C. L.: Assessment of Two-Equation Models for Transonic Flows. AIAA Paper 94-2343, Colorado Springs, Colo., 1994.

14. Menter, F. R.: Two-Equation Eddy-Viscosity Turbulence Models for Engineering Applications. AIAA J., vol. 32, no. 8, 1994, pp. 1598-1605.

15. Townsend, A. A.: Equilibrium Layers and Wall Turbulence. J. Fluid Mech. 11, (97), 1962.

16. Yakhot, V.; Orszag, S. A.; Tangham, S.; Gatski, T. B.; and Speciale, C. G.: Development of Turbulence Models for Shear Flows by a Double Expansion Technique. Phys. Fluids A4 (7), July, 1992, pp. 1510-1520.

17. Menter F. R.: Influence of Freestream Values on $k-w$ Turbulence Model Predictions. AIAA J., vol. 30, no. $6,1992$.

18. Baldwin, B. S.: personal communication, 1993.

19. Durbin, P. A.; Mansour, N. N.; and Yang, Z.: Eddy Viscosity Transport Model for Turbulent Flow. Phys. Fluids, vol. 6, no. 20, Feb. 1994.

20. Spalart, P. R.: personal communication, 1994.

21. Menter, F. R., Performance of Popular Turbulence Models for Attached and Separated Adverse Pressure Gradient Flows. AIAA J., vol. 30, no. 8, Aug. 1992, pp. 2066-2072.

22. Rogers, S. E.: personal communication, 1994.

23. Goldberg, U. C.; and Ramakrishnan, S. V.: A Pointwise Version of the Baldwin-Barth Turbulence Model. International J. Computational Fluid Dynamics 1, no. 4, pp. 321-338.

24. Goldberg, U. C.: A Pointwise One-Equation Turbulence Model for Wall-Bounded and Free Shear Flows. International Symposium on Turbulence, Heat and Mass Transfer, Lisbon, 1994, pp. 13.2.1-13.2.6.
25. Rogers, S. E.; and Kwak. D.: An Upwind Differencing Scheme for the Time-Accurate Incompressible Navier-Stokes Equations. AIAA Paper 882583. Williamsburg, Va. 1988.

26. Rumsey, C. L.; and Vatsa. V. N.: A Comparison of the Predictive Capabilities of Several Turbulence Models Using Upwind and Central-Difference Computer Codes. AIAA Paper 93-0192, Reno, Nev., 1993.

27. Coakley T. J.: personal communication, 1994.

28. Samuel, A. E.; and Joubert. P. N.: A Boundary Layer Developing in an Increasingly Adverse Pressure Gradient. J. Fluid Mech., vol. 66, part 3, 1974, pp. 481-505.

29. Driver, D. M.: Reynolds Shear Stress Measurements in a Separated Boundary Layer. AIAA Paper 91$1787,1991$.

30. Johnson, D. A.; Menter F. R.; and Rumsey, C. L.: The Status of Turbulence Modeling for External Aerodynamics, AIAA Paper-94-2226. Colorado Springs, Colo., 1994.

31. Driver, D. M.; and Seegmiller, H. L.: Features of a Reattaching Turbulent Shear Layer in Divergent Channel Flows. AIAA J., vol. 23, no. 2, 1985.

32. Coles, D.; and Wadcock, A. J.: Flying-Hot-Wire Study of Flow Past a NACA 4412 Airfoil at Maximum Lift. AIAA J., vol. 17, no. 4, 1979.

33. Rogers, S. E.; Wiltberg; N. L.; and Kwak, D.: Efficient Simulation of Incompressible Viscous Flow over Single and Multi-Element Airfoils. AIAA Paper 92-0405, Reno, Nev., 1992.

34. Bachalo, W. D.; and Johnson, D. A.: An Investigation of Transonic Turbulent Boundary Layer Separation Generated on an Axisymmetric Flow Model. AIAA Paper 79-1479, Williamsburg. Va., 1979. 
